\title{
Bu open A retrospective analysis of long-term outcomes following a single episode of transscleral cyclodiode laser treatment in patients with glaucoma
}

\author{
Ivailo Zhekov, ${ }^{1}$ Razia Janjua, ${ }^{1}$ Humma Shahid, ${ }^{1}$ Nicholas Sarkies, ${ }^{1}$ \\ Keith R Martin, ${ }^{1,2}$ Andrew J R White ${ }^{1,2,3}$
}

To cite: Zhekov I, Janjua R, Shahid $\mathrm{H}$, et al. A retrospective analysis of long-term outcomes following a single episode of transscleral cyclodiode laser treatment in patients with glaucoma. BMJ Open 2013;3:e002793

doi:10.1136/bmjopen-2013002793

- Prepublication history for this paper is available online. To view these files please visit the journal online (http://dx.doi.org/10.1136/ bmjopen-2013-002793).

Received 26 February 2013 Revised 27 May 2013 Accepted 29 May 2013

This final article is available for use under the terms of the Creative Commons Attribution Non-Commercial 3.0 Licence; see http://bmjopen.bmj.com

${ }^{1}$ School of Clinical Medicine, Cambridge University Teaching Hospitals NHS Foundation Trust, Cambridge, UK

${ }^{2}$ NIHR Biomedical Research Centre, University of Cambridge, Cambridge, UK ${ }^{3}$ Centre for Vision Research, Westmead Millennium Institute, Sydney, Australia

Correspondence to Dr Ivailo Zhekov; iz209@cam.ac.uk

\section{ABSTRACT}

Objectives: To investigate the efficacy of a single cyclodiode laser photocoagulation treatment for refractory glaucoma and its effect on visual outcome in patients with good visual potential as well as to evaluate possible predictive factors in establishing optimal treatment parameters.

Design: Retrospective observational study.

Setting: Tertiary referral centre.

Participants: The notes of 87 patients with refractory glaucoma who underwent cyclodiode photocoagulation as a first surgical intervention over a 7-year period.

Main outcome measures: Maintenance of intraocular pressure (IOP) reduction, number of medications and visual acuity outcomes post-treatment.

Results: The mean IOP after a single treatment decreased from $39.5 \pm 1.3$ to $17.8 \pm 1.5 \mathrm{~mm} \mathrm{Hg}$ after a 6 -week follow-up period $(p<0.0001)$. This reduction in IOP was maintained over a 3-year period. Here, $61.5 \%$ of patients were able to reduce the number of medications used, with mean reduction from 2.6 to 1.5 medications $(p<0.05)$. The mean initial visual field loss prior to treatment was $8.74 \mathrm{~dB}$ and at 6 months post-treatment was measured at $9.06 \mathrm{~dB}(p>0.05)$, suggesting no significant overall change. Visual acuity remained unchanged or improved for $83.6 \%$ of patients ( $p>0.05$ ) with relatively good visual potential (average vision preoperatively was $0.57 \log M A R$ ). Hypotony occurred in $5.3 \%$ of patients. No patients required enucleation or evisceration.

Conclusions: A single session of cyclodiode laser therapy was associated with significant IOP reduction in a majority of patients with refractory glaucoma. The majority were able to maintain the IOP reduction over a 3-year period without the need for a further surgical intervention. Additionally, over $80 \%$ of the patients in our study were able to maintain their baseline visual acuity. These results support the view that a single cyclodiode treatment can be sufficient in achieving long-term IOP control and may be considered in eyes with relatively good visual potential.

\section{INTRODUCTION}

Transscleral diode laser cyclophotocoagulation (cyclodiode) has been established as a

\section{ARTICLE SUMMARY}

Article focus

- To investigate the efficacy of a single cyclodiode laser photocoagulation treatment for refractory glaucoma.

- To determine the effect of a single cyclodiode laser photocoagulation treatment on visual outcome in patients with good visual potential.

- To evaluate the possible predictive factors in establishing optimal treatment parameters.

Key messages

- A single session of cyclodiode laser therapy was associated with significant intraocular pressure (IOP) reduction in the majority of patients with refractory glaucoma.

- The majority of patients were able to maintain the IOP reduction over a 3-year period without the need for a further surgical intervention.

- Over $80 \%$ of patients in our study were able to maintain their baseline visual acuity.

Strengths and limitations of this study

- The retrospective nature of the study design reflected the need to analyse multiple clinical variables and evaluate different types of outcomes as well as their progression over several years. We aimed to avoid selection bias by including all patients with clearly annotated preoperative and postoperative parameters in the electronic medical record system.

relatively safe and effective intervention for glaucoma. ${ }^{1-6}$ Cyclodiode is often used in refractory glaucoma, where alternative surgical approaches, such as antiproliferative augmented trabeculectomy and tube shunt surgery, may sometimes be judged less appropriate, for example, in eyes with poor visual potential. Additionally, cyclodiode has been shown to be safer than other cyclodestructive procedures, such as Nd:YAG laser cyclophotocoagulation and cyclocryotherapy, which 
present a greater risk of hypotony and phthisis resulting from excessive ciliary body ablation. ${ }^{7-9}$ However, the outcome of cyclodiode therapy is unpredictable and multiple treatments may be required to achieve longterm intraocular control. ${ }^{10} 11$

Currently, there is no general consensus for an optimum treatment protocol for cyclodiode. Data for outcomes following cyclodiode laser treatment vary considerably in the literature. ${ }^{12}$ Parameters such as total delivered energy per eye, number of laser applications per treatment, pulse power, pulse duration and proportion of circumference treated have been analysed with varying results. ${ }^{13-17}$ Furthermore, cyclodiode therapy has often been reserved for eyes with poor vision due to reports that it can lead to a decrease in visual acuity (VA). ${ }^{18}$

The purpose of this study was to evaluate the longterm safety and efficacy of a single episode of transscleral diode laser cyclophotocoagulation for raised intraocular pressure (IOP) in patients with good visual potential at a UK tertiary glaucoma referral centre. The retrospective nature of the study design reflected the need to analyse multiple clinical variables and evaluate different types of outcomes as well as their progression over several years. Additionally, such a study could facilitate the identification predictive factors for optimal treatment parameters by investigating the relation of laser energy delivered and degrees treated to the long-term maintenance in IOP reduction.

\section{METHODS}

The departmental electronic medical record system (Medisoft Ophthalmology, Medisoft Ltd, Leeds, UK) and operating theatre $\log$ books were examined for the period 09/2004-06/2011 to identify patients who had undergone cyclodiode treatment as a first surgical intervention.

All patients were assessed for their response to treatment and those who did not achieve the target IOP reduction were taken as treatment failures. The patients who required either repeat cyclodiode or alternative treatments for IOP control during the follow-up period were also considered as treatment failures from the time of the additional surgical intervention. They were not assessed further for maintenance of postoperative IOP as any additional surgical interventions would interfere with the interpretation of the IOP maintenance data.

Cyclophotocoagulation was performed in the Operating Theatre under subtenon, peribulbar or general anaesthesia using a standard treatment protocol. A lid speculum was placed to achieve adequate exposure for probe placement all around the limbus and a squint hook was used to rotate the globe to achieve exposure in those with narrow palpebral apertures. All treatments were performed using the Oculight $\mathrm{Sx}$ semiconductor diode $810 \mathrm{~nm}$ laser and the contact G Probe (IRIS Medical Instruments, Inc). Standard settings were of
$1500 \mathrm{~ms}$ duration and $1500 \mathrm{~mW}$ power. The energy levels were not routinely titrated for 'pops' and the power settings were left at $1500 \mathrm{~mW}$. Transillumination was performed for ciliary body identification. Usually 10 applications were applied per quadrant for $180-360^{\circ}$, with the applications spaced approximately one-half width of the probe tip apart. The 3 and 9 o'clock positions were avoided to spare the long ciliary nerves. Postoperative steroid drops were used for 4 weeks. Glaucoma medications were continued after cyclodiode treatment and adjusted later as required.

Success was defined as an IOP of $6-21 \mathrm{~mm} \mathrm{Hg}$ at the last follow-up visit without the need for oral acetazolamide and an IOP reduction of at least $30 \%$ compared with pretreatment. Hypotony was defined as an IOP of $5 \mathrm{~mm} \mathrm{Hg}$ or less. The laser energy used was classified as either low energy (median value $45 \pm 11.47 \mathrm{~J}$, range 22.5 $67.5 \mathrm{~J}$ ) or high energy (median value $90 \pm 13.42 \mathrm{~J}$, range $67.5-130 \mathrm{~J}$ ). The follow-up period in IOP measurements was 3 years. VA prior to treatment was assessed for all patients using the LogMAR scale. Visual field testing was performed with the Humphrey Visual Field Analyser using the SITA 24-2 threshold programme. Mean deviation (MD) of visual field sensitivity loss in decibels was taken as our surrogate measure of visual field loss.

Data were analysed using Microsoft Excel and, where appropriate, Student's t test was used to calculate $p$ values. $R^{2}$ values were derived from linear regression analyses using the same programme. The Kaplan-Meier 'survival' analysis was performed using GraphPad Prism 5.0. Approval for the audit was granted by the Cambridge University Hospitals NHS Foundation Trust Ethics Committee. The tenets of the Declaration of Helsinki were observed.

\section{RESULTS}

From our records, we were able to identify 104 patients who underwent cyclodiode therapy as a first surgical treatment modality in the period from 09/2004 to 06/ 2011 at Addenbrooke's Hospital, Cambridge. Of these, complete medical records were available for 87 patients with follow-up data available for up to 3 years. Preoperative patient data are summarised in table 1.

\section{Change in IOP}

The mean IOP decreased significantly from $39.5 \mathrm{~mm} \mathrm{Hg}$ (SE 1.26) before cyclodiode therapy to $17.8 \mathrm{~mm} \mathrm{Hg}$ (SE $1.51)$ at 6 weeks post-treatment, an observable reduction of $45.1 \% \quad(p<0.0001)$. There was a mild linear correlation between preoperative IOP and observed IOP reduction $\left(\mathrm{R}^{2}=0.32\right.$; figure $\left.1 \mathrm{~A}\right)$.

\section{Safety and efficacy of cyclodiode treatment}

An IOP reduction greater than $30 \%$ of initial IOP was achieved at 6 weeks in $67.7 \%$ of patients, while hypotony occurred in only five cases or $5.3 \%$ of patients $(p<0.05)$. None of the patients experienced post-treatment uveitis 
Table 1 Baseline demographics of the patients and clinical parameters including diagnostic features, preoperative IOP and visual acuity assessments

\begin{tabular}{ll}
\hline Category & Cases $(\mathbf{n = 8 7})$ \\
\hline Mean age (years) & 66.3 \\
Sex (n (\%)) & $49(56.3)$ \\
$\quad$ Male & $38(43.7)$ \\
$\quad$ Female & 0.57 LogMAR \\
Preoperative VA (mean) & $39.5 \pm 1.3 \mathrm{~mm} \mathrm{Hg}$ \\
IOP (mm Hg), mean (SD) & 2.6 \\
Mean number of glaucoma medications & \\
Glaucoma type (n (\%)) & $33(37.9)$ \\
$\quad$ Primary open angle glaucoma (POAG) & $6(6.9)$ \\
$\quad$ Primary angle closure glaucoma & \\
$\quad$ (PACG) & $25(28.7)$ \\
$\quad$ Neovascular glaucoma & $5(5.7)$ \\
$\quad$ Uveitic glaucoma & $18(20.8)$ \\
Secondary open angle glaucoma & \\
(SOAG) - other & \\
\hline IOP, intraocular pressure; VA, visual acuity. &
\end{tabular}

or required further treatment or enucleation for pain symptoms (figure 1B). The data for the patients who developed hypotony such as initial diagnosis, treatment settings, IOP measurements and VA outcome are summarised in table 2.

\section{Long-term maintenance of IOP reduction after a single} cyclodiode treatment

The IOP reduction was maintained for a long term over a period of 3 years in the majority of our patients. Measurements were taken preoperatively (39.5 $\pm 1.3 \mathrm{~mm} \mathrm{Hg}$ ) as well as postoperatively at 6 weeks (17.8 $\pm 1.5 \mathrm{~mm} \mathrm{Hg})$ and 6 months $(19.6 \pm 1.5 \mathrm{~mm} \mathrm{Hg})$ for all patients (figure 1C). Patients who required additional procedures in that eye post-treatment were excluded from further analysis from the time point of additional intervention as it would artificially lower their IOP measurements The follow-up measurements at 1 (18.9 mm Hg), $2(22.1 \mathrm{~mm} \mathrm{Hg})$ and 3 years $(21.7 \mathrm{~mm} \mathrm{Hg})$ were all after a single cyclodiode treatment.

\section{Kaplan-Meier analysis of IOP reduction 'survival'} following a single cyclodiode treatment

Success was defined as an IOP of 6-21 $\mathrm{mm} \mathrm{Hg}$ at the last follow-up visit without the need for oral acetazolamide and an IOP reduction of at least $30 \%$ compared with preoperatively (figure 2). The proportion of patients to maintain the desired IOP reduction after a single cyclodiode treatment without the need for further IOP-lowering intervention in our study was $67.7 \%$ at 6 weeks postoperatively, $66.2 \%$ at 6 months, $63 \%$ at 1 year, $61.2 \%$ at 2 years and $61.2 \%$ at 3 years. Of the patients who responded to treatment at 6 weeks, $90.4 \%$ were able to maintain the IOP reduction over a period of 3 years.
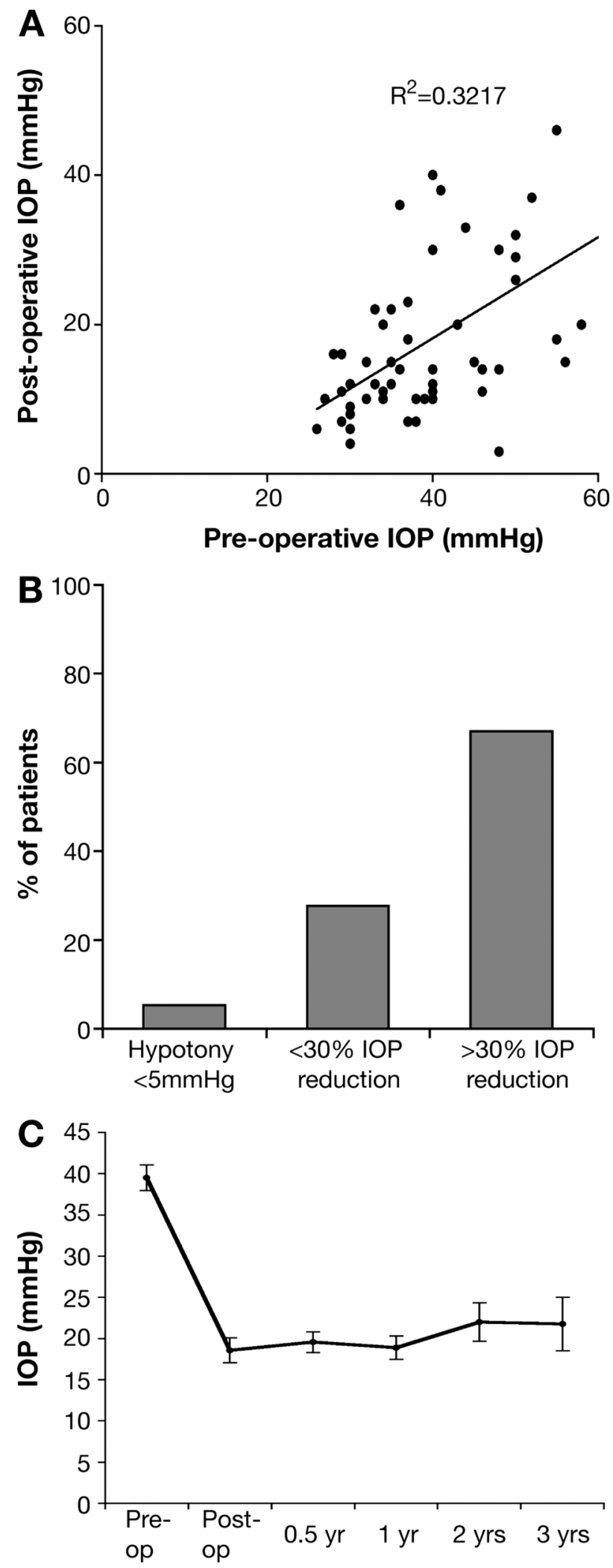

Time at measurement

Figure 1 (A) Change in intraocular pressure (IOP) following a single cyclodiode treatment. (B) Safety and efficacy of cyclodiode treatment. (C) IOP reduction was maintained long term over a period of 3 years after single treatment. 
Table 2 Details of patients who developed hypotony including diagnostic and treatment features, peri-operative IOP assessments and duration of hypotony

\begin{tabular}{|c|c|c|c|c|c|c|c|c|c|}
\hline & Diagnosis & $\begin{array}{l}\text { Energy } \\
\text { used }\end{array}$ & $\begin{array}{l}\text { Degrees } \\
\text { treated }\end{array}$ & $\begin{array}{l}\text { Peak IOP } \\
(\mathrm{mm} \mathrm{Hg})\end{array}$ & $\begin{array}{l}\text { Postop } \\
\text { IOP } \\
(\mathrm{mm} \mathrm{Hg})\end{array}$ & $\begin{array}{l}\text { Hypotony } \\
\text { duration } \\
\text { (months) }\end{array}$ & $\begin{array}{l}\text { Last IOP } \\
(\mathrm{mm} \mathrm{Hg})\end{array}$ & $\begin{array}{l}\text { Preop VA } \\
\text { (LogMAR) }\end{array}$ & $\begin{array}{l}\text { Last VA } \\
\text { (LogMAR) }\end{array}$ \\
\hline PT1 & Neovascular & $90 \mathrm{~J}$ & 180 & 58 & 2 & 6 & 10 & 0.78 & $\mathrm{PL}$ \\
\hline PT2 & Neovascular & $90 \mathrm{~J}$ & 360 & 41 & 4 & 6 & 32 & PL & NPL \\
\hline РT3 & Neovascular & 120J & 360 & 40 & 2 & 6 & 9 & 0.6 & $\mathrm{HM}$ \\
\hline PT4 & SOAG (other) & $120 \mathrm{~J}$ & 360 & 26 & 3 & 6 & 8 & 1.0 & $\mathrm{CF}$ \\
\hline PT5 & SOAG (other) & $120 \mathrm{~J}$ & 360 & 48 & 2 & - & 3 & $\mathrm{HM}$ & $\mathrm{HM}$ \\
\hline
\end{tabular}

\section{Cyclodiode effect on glaucoma medications}

A significant proportion of the patients $(61.5 \%)$ were able to decrease the number of medications they are taking for IOP control following cyclodiode treatment (figure 3A). A decrease of 2 medications or more was achieved by $34.6 \%$ of patients, while $26.9 \%$ of patients decreased their medication by one. Overall, the average number of medications decreased from 2.6 before cyclodiode treatment to 1.5 after cyclodiode treatment $(\mathrm{p}<0.05)$.

\section{Change in VA}

A single episode of cyclodiode treatment had no significant effect on VA. The mean VA measurements changed from $0.5720 \mathrm{Log}$ MAR units before cyclodiode therapy to 0.5408 Log MAR units post-treatment, which was not statistically significant $(p>0.05$; figure $3 \mathrm{~B})$. The data analysis was performed on 47 patients $(54.1 \%)$ who had clearly annotated numerical values for their VA measurements both preoperatively and postoperatively. The boxed numerals in the graph represent the number of values at the same point.

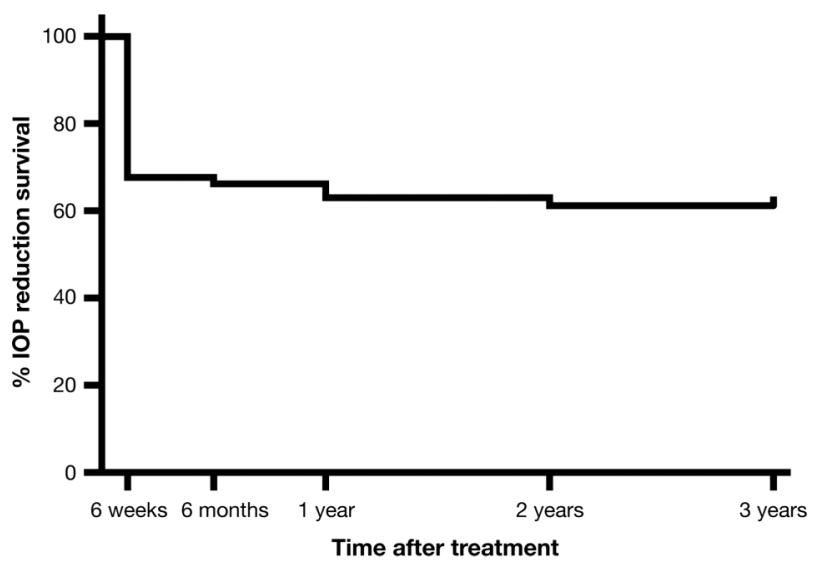

Figure 2 Kaplan-Meier analysis for a cumulative proportion of success in IOP control after a single cyclodiode treatment. Survival was defined as a sustained intraocular pressure (IOP) drop of at least $30 \%$ compared with pretreatment or sustained IOP of $6-21 \mathrm{~mm} \mathrm{Hg}$ without the need for additional IOP-lowering medications or further surgical interventions.

\section{Change in visual fields}

The average visual field sensitivity remained almost unchanged with an average MD value of $-8.74 \mathrm{~dB}$ pretreatment compared to the MD value of $-9.05 \mathrm{~dB}$ at 6 months post-treatment, which was not statistically significant $(p>0.05)$. The data analysis was performed on 36 patients $(41.3 \%)$ who had clearly annotated numerical values for their visual fields measurements both preoperatively and postoperatively (figure $3 \mathrm{C}$ ).

\section{Effect of laser energy on IOP reduction}

Of the patients who received high-energy treatment $(90 \mathrm{~J}), 80.3 \%$ obtained pressure reduction of $>30 \%$ of initial IOP compared with $56.8 \%$ in patients who received low-energy treatment $(45 \mathrm{~J}$ ) (figure $4 \mathrm{~A}$ ).

\section{Effect of laser energy on VA}

Of the patients who received high-energy treatment $(90 \mathrm{~J}), 18.6 \%$ noted improvement equivalent to at least one Snellen line compared with $6.7 \%$ in patients who received low-energy treatment (figure $4 \mathrm{~B}$ ).

\section{Effect of degrees treated on IOP reduction}

Of the patients who underwent $360^{\circ}$ treatment, $71.4 \%$ obtained pressure reduction greater than $30 \%$ of initial IOP compared with $55 \%$ of patients who underwent $180^{\circ}$ treatment (figure $4 \mathrm{C}$ ).

\section{Effect of degrees treated on VA}

Of the patients who underwent $360^{\circ}$ treatment, $20.9 \%$ noted improvement equivalent to at least one Snellen line compared with $5.5 \%$ of patients who underwent $180^{\circ}$ treatment (figure $4 \mathrm{D}$ ).

\section{DISCUSSION}

The results of this retrospective analysis are supportive of a single cyclodiode treatment being effective in the management of refractory glaucoma. Although this is a retrospective rather than a prospective study, we aimed to avoid selection bias by including all patients either as success or failure of treatment. The patients who required additional surgical interventions were taken as failure from the time of additional intervention. 

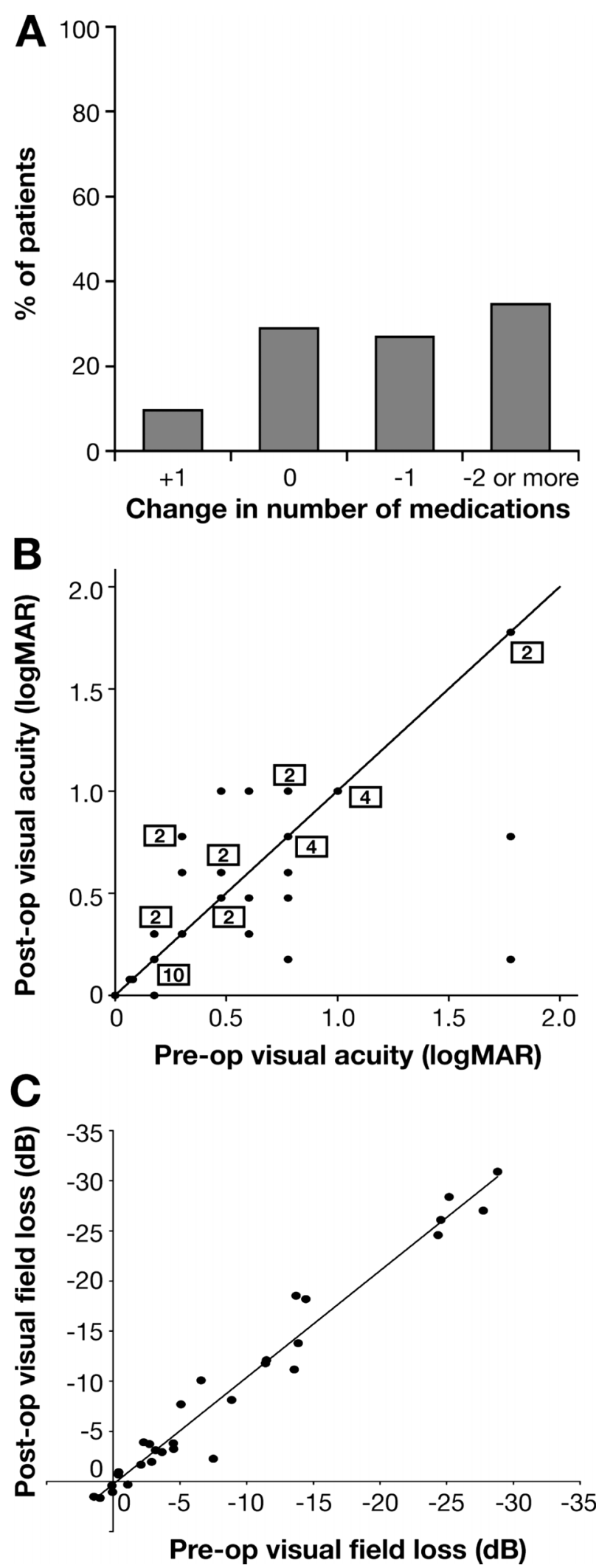

Figure 3 (A) Cyclodiode effect on antiglaucoma medications. (B) Preoperative and postoperative visual acuity measurements in LogMAR units after a single cyclodiode treatment. The numbers in squares annotate the numbers of patients with the same values on the $x$ and $y$ axes. (C) Preoperative and postoperative visual field loss taken from the MD values $(\mathrm{dB})$ on the Humphrey Visual Field analyser after a single cyclodiode treatment.
Our data show that $67.7 \%$ of patients achieved an IOP reduction greater than $30 \%$ of presenting IOP at 6 weeks and $61.5 \%$ could reduce the number of medications by at least 1 . Hypotony appears to be related to the type of glaucoma (with neovascular glaucoma being associated with worse outcome) as well as to the number of degrees treated and total energy used. Indeed, the patients with neovascular glaucoma in our cohort were almost four times more likely to develop hypotony compared to any other types of glaucoma.

In our patients, the mean IOP decreased significantly from $39.5 \mathrm{~mm} \mathrm{Hg}$ (SE 1.26) before cyclodiode therapy to $17.8 \mathrm{~mm} \mathrm{Hg}$ (SE 1.51) at 6 weeks post-treatment, an observable reduction of $45.1 \%(\mathrm{p}<0.05)$. The results of the linear regression analysis indicate that there might be some predictive value in determining IOP reduction based on initial IOP measurements such as the likelihood of the greatest reduction in IOP being seen in patients with the highest IOP at the time of treatment $\left(\mathrm{R}^{2}=0.32\right)$. A similar relationship was reported by Vernon et $a l,{ }^{19}$ where eyes with IOP greater than $30 \mathrm{~mm} \mathrm{Hg}$ were more likely to exhibit a pressure drop greater than $30 \%$ of initial IOP.

The IOP reduction was maintained long term in $61.2 \%$ of all patients in our study after a single cyclodiode treatment. Of the patients who responded to treatment at 6 weeks, $90.4 \%$ were able to maintain the IOP reduction over a period of 3 years. Similar sustainability of the mean IOP reduction postoperatively is also reported by Bloom et $a l^{18}$ and Kosoko et al, ${ }^{20}$ although in both these studies the follow-up was considerably shorter, the average time being less than 2 years. The results from our patient cohort suggest that a single session of cyclodiode therapy could potentially be a viable initial treatment modality for maintaining a longer term IOP control in patients with refractory glaucoma.

In our study, of the patients who received high-energy treatment $(90 \mathrm{~J}), 80.3 \%$ obtained an IOP reduction of greater than $30 \%$ of the initial IOP compared with $56.8 \%$ in patients receiving low-energy treatment $(45 \mathrm{~J})$. This is consistent with a meta-analysis by Iliev et $a l^{17}$ where a comparison of different laser protocols from different studies suggested that the use of a low-energy protocol could potentially result in the lowest IOP reduction, the highest postoperative IOP and the highest retreatment rate overall. We also observed a similar relationship when patients were assessed in regard to the degrees treated rather than the energy used. Here, of the patients who underwent $360^{\circ}$ treatment, $71.4 \%$ obtained pressure reduction greater than $30 \%$ of initial IOP compared with $55 \%$ of patients who underwent $180^{\circ}$ treatment.

Hypotony was the most common long-term complication in our study $(5.3 \%)$, which is in accordance with other published reports. ${ }^{14}$ In our cohort, the incidence of hypotony was much more likely with an eye with neovascular glaucoma (table 2). Murphy et $a l^{14}$ also found a 
A
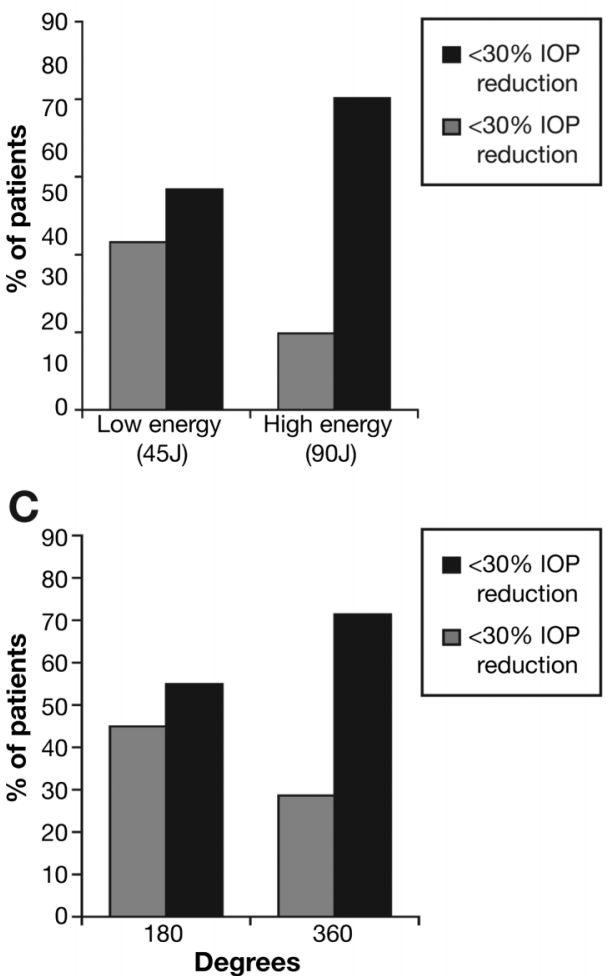

B

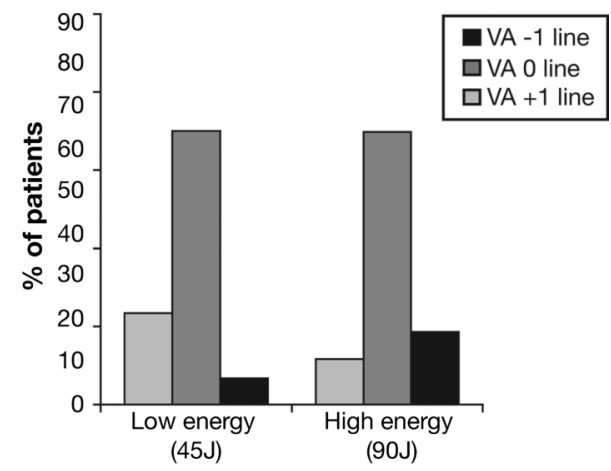

D

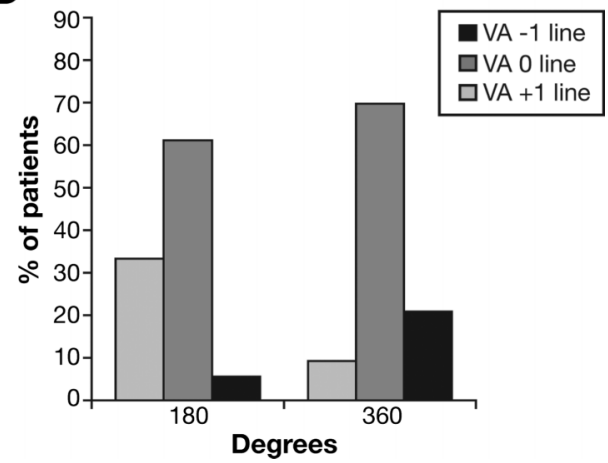

Figure 4 (A) Effect of laser energy on intraocular pressure (IOP) reduction. (B) Effect of laser energy on visual acuity (VA). (C) Effect of degrees treated on IOP reduction (D) Effect of degrees treated on VA.

higher risk of hypotony in eyes with neovascular glaucoma, particularly if the preoperative IOP was high and the energy level used was greater. This correlation between hypotony and higher energy used in cyclodiode is also observed in several other studies. ${ }^{12}$ However, univariate regression analysis performed by Murphy et $a l^{14}$ shows that very high pretreatment IOP alone, possibly causing ciliary body ischaemia, could be responsible for the high incidence of hypotony in patients receiving high-energy treatment in their study.

Additionally, in a retrospective analysis of 209 eyes by Bloom et $a l,{ }^{18}$ a very low incidence of hypotony $(1 \%)$ was observed with a high-energy protocol when the mean energy used was $90 \mathrm{~J}$. Furthermore, Iliev et al ${ }^{17}$ report in their study that eyes developing hypotony had not received higher energies compared with eyes that maintained normal IOP. Therefore, the intention to reduce hypotony risk by using a lower laser power and fewer applications per treatment should be weighed against the possibility of a lower efficacy of the cyclodiode treatment.

VA remained unchanged for the majority of our patients who underwent cyclodiode treatment $(69.9 \%)$. In our study, $16.4 \%$ reported deterioration in VA of one Snellen line, while $13.7 \%$ of the patients reported an improvement in VA of one Snellen line after 6 weeks. The latter probably resulted either from resolution of preoperative corneal oedema or inter-observer variation in VA testing. Similar results are also reported in a study by Murphy et al, ${ }^{14}$ where VA remained the same in
$74.6 \%$ of patients at 6 months following cyclodiode treatment. In our study, most patients with difficult to manage glaucoma retained their good VA in the long term following cyclodiode treatment. The proportion losing two Snellen lines is actually better than that reported after trabeculectomy or tube surgery. ${ }^{21}$ These results suggest a possible role for the use of transscleral cyclodiode treatment in eyes with relatively good visual potential; however, further controlled prospective studies would be required to better define this role.

This study is the first to measure MD values for the patients both preoperatively and postoperatively in order to provide a more objective assessment of glaucoma-related visual loss. The MD values in our patients remained virtually unchanged after cyclodiode treatment from $-8.74 \mathrm{~dB}$ preoperatively to $-9.05 \mathrm{~dB}$ postoperatively. Previously, it has been reported that the most frequent cause of visual loss was further progression of glaucoma, which was an attributable cause in over half of the cases. ${ }^{22}$ The peri-operative visual field assessment presented in this study suggests that the maintenance of VA observed in the majority of the patients following cyclodiode treatment could be due to a good postoperative IOP control. There are no previous retrospective or prospective studies evaluating visual field measurements perioperatively in patients undergoing cyclodiode treatment and this most likely reflects the difficulties associated with performing the tests in patients with poor VA. Our study therefore is the first to provide a standardised measurable evidence of maintenance of patients' visual fields as a 
marker of glaucoma progression following a single cyclodiode treatment. Our study's main limitations are its retrospective nature and the absence of a control group to distinguish the adverse effects of the treatment from the natural history of the underlying disease.

The results of this study suggest that the IOP reduction after cyclodiode treatment could potentially prevent further deterioration in the vision of patients with glaucoma. Currently, according to the UK National Cyclodiode Survey Study, only $60 \%$ of practitioners would perform cyclodiode procedure in the presence of good VA. ${ }^{12}$ Until recently, cyclodiode treatment has been associated with subsequent loss of VA in a significant percentage of the patients. ${ }^{18} 2324$ However, cyclodiode use in eyes with useful vision has already been proposed in several studies. ${ }^{192-25}$ Furthermore, the risk of VA loss in patients after multiple cyclodiode treatments does not appear to be any greater than that after any other surgical modality used for treating patients with glaucoma as reported in a study by Rotchford $e t a l^{24}$

In our study, the better outcome in VA was most pronounced in the high-energy group, probably due to the more effective IOP control. Of the patients who received high-energy treatment $(90 \mathrm{~J}), 18.6 \%$ noted improvement of at least one Snellen line compared with $6.7 \%$ in patients who received low-energy treatment (45J). Similar results are observed when patients were assessed in regard to the degrees treated. Here, of the patients who underwent $360^{\circ}$ treatment, $20.9 \%$ noted improvement equivalent to at least one Snellen line compared with $5.5 \%$ of the patients who underwent $180^{\circ}$ treatment.

In conclusion, conventional diode cyclophotocoagulation is characterised by low incidence of complications and therefore appears to be a safe and effective treatment for refractory glaucoma. In our study, the IOP pressure was effectively reduced in patients with glaucoma after a single cyclodiode treatment without adverse effects on VA in the majority of the patients over a 3-year period. Hypotony seems to be the main risk of treatment and could be limited by reducing the laser energy applied to less than $90 \mathrm{~J}$, particularly in patients with neovascular glaucoma.

Contributors IZ, RJ, KRM and AJRW conceived the idea of the study and were responsible for the design of the study. IZ, HS, KRM and AJRW were responsible for undertaking the data analysis and produced the tables and graphs. RJ and NS provided input into the data analysis. The initial draft of the manuscript was prepared by IZ, HS, KRM and AJRW and then circulated repeatedly among all authors for critical revision. IZ and RJ were responsible for the acquisition of the data, and IZ, RJ, HS, NS, KRM and AJRW contributed to the interpretation of the results. All authors read and approved the final manuscript.

Funding This research received no specific grant from any funding agency in the public, commercial or not-for-profit sectors.

Competing interests None.

Ethics approval Cambridge University Hospitals NHS Foundation Trust Ethics Committee.

Provenance and peer review Not commissioned; externally peer reviewed.

Data sharing statement No additional data are available.

\section{REFERENCES}

1. Hennis HL, Stewart WC. Semiconductor diode laser transscleral cyclophotocoagulation in patients with glaucoma. Am J Ophthalmol 1992;113:81-5.

2. Hawkins TA, Stewart WC. One-year results of semiconductor transscleral cyclophotocoagulation in patients with glaucoma. Arch Ophthalmol 1993;111:488-91.

3. Brancato R, Carassa RG, Bettin P. Contact transscleral cyclophotocoagulation with diode laser in refractory glaucoma. Eur $J$ Ophthalmol 1995;5:32-9.

4. Threlkeld $\mathrm{AB}$, Johnson $\mathrm{MH}$. Contact transscleral diode cyclophotocoagulation for refractory glaucoma. J Glaucoma 1999;8:3-7.

5. Egbert PR, Fiadoyor S, Budenz DL. Diode laser transscleral cyclophotocoagulation as a primary surgical treatment for primary open angle glaucoma. Arch Ophthalmol 2001;119:345-50.

6. Mistlberger A, Liebmann JM, Tschiderer H. Diode laser transsclera cyclophotocoagulation for refractory glaucoma. J Glaucoma 2001;10:288-93.

7. Benson MT, Nelson ME. Cyclocryotherapy: a review of cases over a 10-year period. Br J Ophthalmol 1990;74:103-5.

8. Schuman JS, Bellows AR, Shingleton BJ. Contact transscleral Nd: YAG laser cyclophotocoagulation. Ophthalmology 1992;99: 1089-94.

9. Ulbig MW, McHugh DA, McNaught Al. Clinical comparison of semiconductor diode versus neodymium:YAG non-contact cyclophotocoagulation. Br J Ophthalmol 1995;79:569-74.

10. Pucci V, Tappainer F, Borin S, et al. Long term follow up after transscleral diode laser photocoagulation in refractory glaucoma. Ophthalmologica 2003;217:279-83.

11. Grueb M, Rohrbach JM, Bartz KU, et al. Transscleral diode laser cyclophotocoagulation as primary and secondary surgical treatment in primary open angle and pseudoexfoliatve glaucoma: long term clinical outcomes. Graefes Arch Clin Exp Ophthalmol 2006;244:1293-9.

12. Agrawal P, Dulku S, Nolan W, et al. The UK National Cyclodiode Laser Survey. Eye 2011;25:168-73.

13. Walland MJ. Diode laser cyclophotocoagulation: longer term follow up of a standardized treatment protocol. Clin Exp Ophthalmol 2000;28:263-7.

14. Murphy CC, Burnett CAM, Spry PDG, et al. A two centre study of the dose-response relation for transscleral diode laser cyclophotocoagulation in refractory glaucoma. $\mathrm{Br} J$ Ophthalmol 2003;87:1252-7.

15. Chang SH, Chen YC, Li CY. Contact diode laser transscleral cyclophotocoagulation for refractory glaucoma: comparison of two treatment protocols. Can J Ophthalmol 2004;39:511-16.

16. Noureddin BN, Zein W, Haddad C. Diode laser transcleral cyclophotocoagulation for refractory glaucoma: a 1 year follow-up of patients treated using an aggressive protocol. Eye 2006;20: 329-35.

17. Iliev ME, Gerber S. Long-term outcome of trans-scleral diode laser cyclophotocoagulation in refractory glaucoma. $\mathrm{Br} J$ Ophthalmol 2007:91:1631-5.

18. Bloom PA, Tsai JC, Sharma K, et al. Cyclodiode trans-scleral diode laser cyclophotocoagulation in the treatment of advanced refractory glaucoma. Ophthalmology 1997;104:1508-20.

19. Vernon SA, Koppens JM, Menon GJ, et al. Diode laser cycloablation in adult glaucoma:long-term results of a standard protocol and review of current literature. Clin Exp Ophthalmol 2006;34:411-20.

20. Kosoko O, Gaasterland DE, Pollack IP. Long-term outcome of initial ciliary ablation with contact diode laser transscleral cyclophotocoagulation for severe glaucoma. The diode laser ciliary ablation study group. Ophthalmology 1996;103:1294-302.

21. Gedde SJ, Schiffman JC, Feuer WJ, et al. Treatment outcomes in the tube versus trabeculectomy study after one year of follow-up. Am J Ophthalmol 2007;143:9-22.

22. Rotchford AP, Jayasawal R, Madhusudhan S, et al. Transscleral diode laser cycloablation in patients with good vision. $\mathrm{Br} J$ Ophthalmol 2010;94:1180-3.

23. Youn J, Cox TA, Allingham RR. Factors associated with visual acuity loss after noncontact transscleral Nd:YAG cyclophotocoagulation. J Glaucoma 1996;5:390-94.

24. Wilensky JT, Kammer J. Long-term visual outcome of transscleral laser cyclotherapy in eyes with ambulatory vision. Ophthalmology 2004;111:1389-92.

25. Ansari E, Gandhewar J. Long-term efficacy and visual acuity following transscleral diode laser photocoagulation in cases of refractory and non-refractory glaucoma. Eye 2007;21: 936-40. 